\title{
Design of Temperature and Humidity Monitoring System of Environmental Chamber Based on ZigBee and SHT11
}

\author{
Ning Guo ${ }^{1, a, *}$, Qiong Zhang ${ }^{1, b}$, Tao Wang ${ }^{1, c}$ and Chuang Shen ${ }^{1, d}$ \\ ${ }^{1}$ Shijiazhuang New Technology Application Institute, Shijiazhuang 050000, China \\ amhwl_gn_lmyy@163.com, b66902352@qq.com, c372797121@qq.com, ${ }^{\text {d} 443588769 @ q q . c o m ~}$ \\ *corresponding author
}

Keywords: ZigBee, SHT11, Temperature and humidity, Monitoring

\begin{abstract}
In view of problems existing in data acquisition and monitoring methods for environmental chamber test, this paper design a temperature and humidity monitoring system based on ZigBee and SHT11. The CC2530 RF as the core is used to form the wireless network. The sensor nodes carrying the SHT11 transmit temperature and humidity parameters to the coordinator and then puts forward it to the mobile terminal. The coordinator can record, display, store and process the received data in real time and send the alert signal. And the mobile terminal can set and query the information. The testing results indicate that the measurement accuracy is high, the data transmission is stable and reliable, and the mobile terminal achieves the temperature and humidity data acquisition and monitoring flexibly.
\end{abstract}

\section{Introduction}

Temperature and humidity environmental test as a means of verifying the performance of electronic equipment, exposing defects in the design and process, and improving reliability of products, is widely applied to product development, production and use. The traditional data recording and equipment monitoring methods are inefficient, and the data is not continuous, the number of device sensors is small which influences the accuracy of the test greatly, and affects the reliable estimate of electronic equipment to the environment. In addition, for wired acquisition and monitoring system, it has some defects, such as complex cabling, high cost, limited monitoring and intractable to maintenance. Adopting wireless system to acquire data and monitor equipment becomes the main research direction to solve such problems.

ZigBee is a short-range, low-power wireless communication technology. With its low complexity, low consumption, and self-organization, ZigBee is widely applied in the field of automatic control and remote control ${ }^{[1,2]}$. Based on its specialities, this paper designs a wireless temperature and humidity monitoring system with low wiring, simple schematic and low cost, which realizes the real-time acquisition, wireless transmission and overrun alarm of alternating environmental chamber, and accomplishes an intended result.

\section{General Design of the system}

The temperature and humidity monitoring system of environmental chamber based on ZigBee and SHT11 achieves the temperature and humidity data automatically acquired, wireless transmission and overrun alarm. The system is composed of three parts: sensor nodes, coordinator and handheld terminal, and communicates through ZigBee network. The sensor nodes are distributed on the chamber, and the temperature and humidity sensors SHT11 on the node acquire the data inside and outside of the chamber. The sensors in the box are arranged per the environmental chamber. The coordinator is used for the establishment and maintenance of the ZigBee wireless network. In addition, it summarizes the data, analyzes, stores and selectively forwards to handheld terminal. And for handheld terminal, it is a mobile monitoring terminal with real-time view of the acquired temperature and humidity information and alarm threshold setting. 
What's more it receives the alarm signal once the temperature and humidity overrun. The whole system schematic shown in Fig.1.

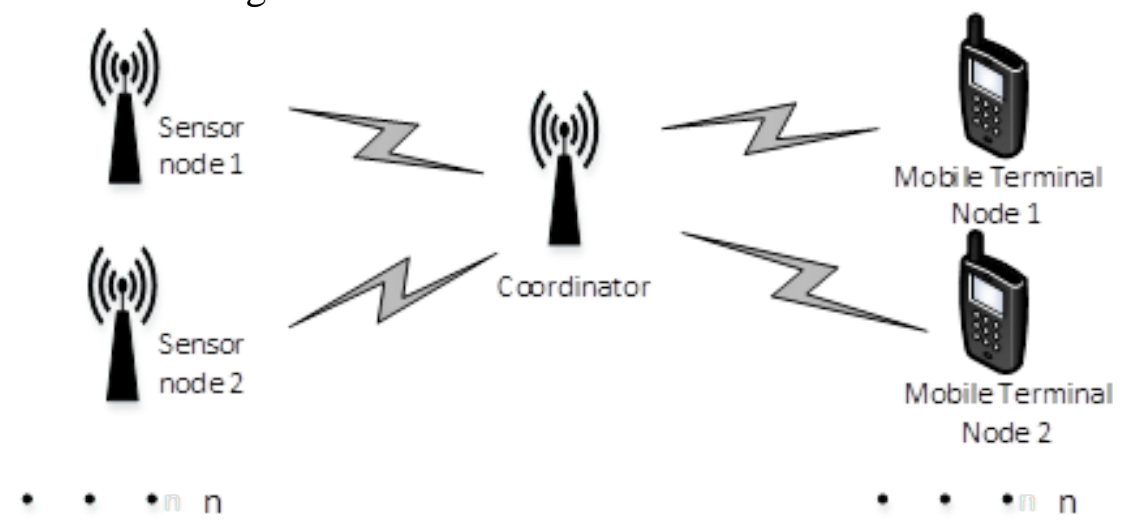

Fig. 1 The schematic of whole system

\section{Hardware Design}

The coordinators, mobile terminals, sensor nodes in this system are developed with chip CC2530 of TI which is satisfied standard ZigBee agreement. The system uses the enhanced 8051 microcontroller with $128 \mathrm{~KB}$ of programmable Flash, 8KB of RAM, 18 interrupt sources, two Uart serial transceivers, and 7-12 bit resolution analog-to-digital converters (ADCs) and satisfies the requirements of low power, low cost, high efficiency. Each module is independently designed the peripheral system circuit according to its function.

\subsection{Sensor Node Design}

The sensor node mainly consists of CC2530 module, temperature and humidity sensor (SHT11), power supply module. And its hardware schematic is shown in Fig.2. The main function of the sensor node is acquiring the temperature and humidity data inside and outside of the chamber and sending it to the coordinator.

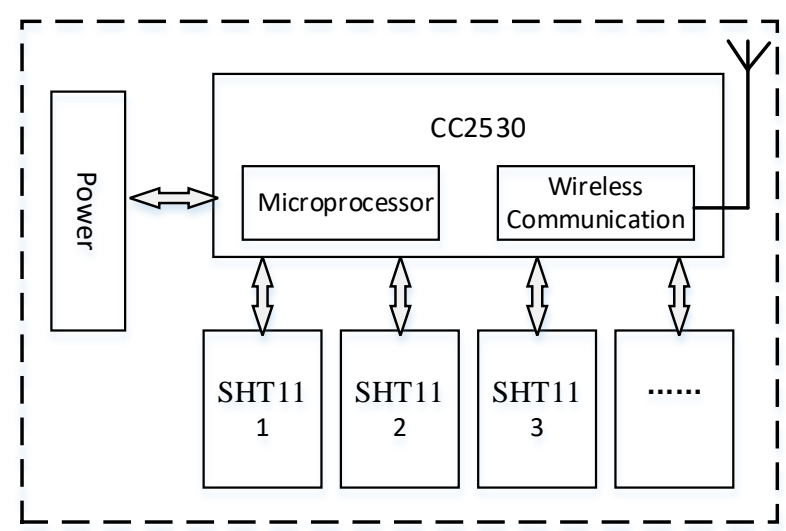

Fig.2 The schematic of sensor node

In Figure 2, the digital temperature and humidity sensor SHT11 with high-precision is developed by the Swiss company Sensirion designed with CMOSens ${ }^{\circledR}$ technology. And it has high reliability and excellent stability. This chip contains a temperature sensing element, a moisture sensitive element, and a 14-bit A / D converter . The sensor module is connected to the CC2530 through two wires, where needs a 10K pull-up resistor between the DATA pin of SHT11 and power supply. In addition, there is a 100nF capacitor attached to VCC and GND pin to ensure accurate and effective signal transmission. The CC2530 of the sensor node, after receiving the data acquired by the sensor, sends the data to the coordinator via the built-in RF transceiver. 


\subsection{Coordinator Design}

The coordinator is used to start and configure the network, and expands the network communication range, assists the sensor nodes in the network and mobile terminals communicate with each other. In addition, it is the centralized collector and data storage of the entire wireless network, and the schematic of this node is shown in Fig.3.

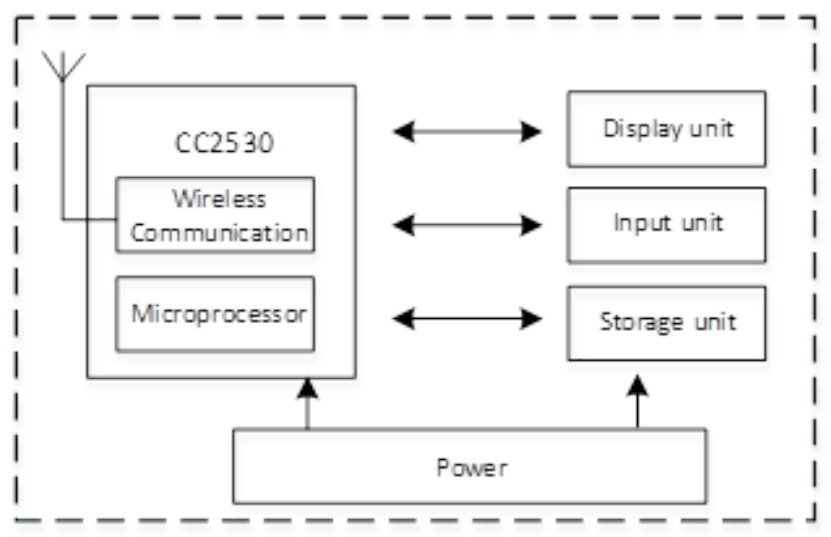

Fig.3 The schematic of coordinator node

Coordinator and sensor node structure are similar, and both take CC2530 as the main control module integrated with data processing and wireless communication functions. This module combines wireless transmission, storage, display and keying, and it constitutes a highly efficient forwarding mechanism.

\subsection{Mobile Terminal Node Design}

Mobile terminal is a visual "mobile alarm" and "query machine" that supplies a real-time view of the temperature and humidity information of the nodes and terminal around, and can set the alarm threshold for each node. The schematic is shown in Figure 4.

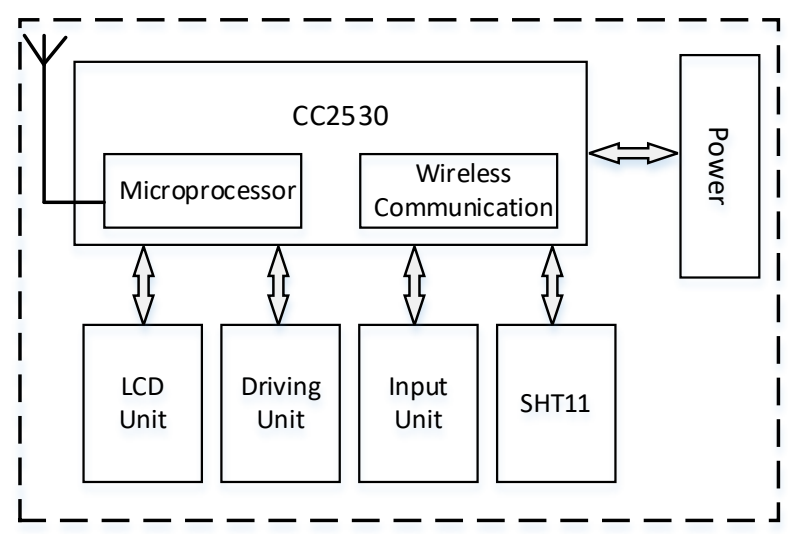

Fig.4 The schematic of mobile terminal

The mobile terminal can monitor the sensor nodes within the limited communication capacity of the entire wireless network, and its advantages are mainly mobility. The monitoring host computer is set in the fixed distance from the test equipment, and when the operator monitors test, there is limited area and it is affected by the experimental equipment, such as noise and other environmental factors. The set of mobile terminal make a normal test data recording and a real-time alarm signal finding, and also mitigates the constraints of the test equipment and site.

\section{Software Design}

In order to ensure the effective storage of the data, all mobile terminals and sensor nodes are transmitted through the coordinator. 
The sensor nodes are located on the bottom of the wireless network. And they basically fulfill the same tasks that monitoring data acquisition and transmission, and responding to the information received in the coordinator.

The coordinator is a link between the monitoring nodes and the handheld terminals, which mainly processes and forwards the data receiving from the sensors. For this network with fixed nodes, in order to reduce the complexity of programming and ensure the efficient operation of the system, the coordinator program sets unique addresses of the sensors and mobile terminals, and if system adds a new equipment into the network, only fine tuning the coordinator program.

The functions of mobile terminal node are query, monitoring data, and it is a mobile human-computer interaction monitoring tool. And the interface design of the mobile terminal is fully digital, text, no curve, graphics or other complex interface that ensure covering all requirements and reduce the complexity of programming as possible.

\section{Conclusion}

In this paper, ZigBee and SHT11 sensor are adopted to achieve the design of wireless monitoring system of temperature and humidity data of environmental chamber. And this system is verified by testing. By the test, in this system, the sensors are arranged evenly and the temperature and humidity data are acquired accurately. The mobile terminals are flexible, simple and practical, accurate records, display data comprehensively, and satisfy the requirements of test monitoring. What's more the system saves the test manpower, material and financial resources with its small wiring, strong mobility and low maintenance cost. The innovation of sensors' location setting not only meets the temperature and humidity data acquisition, but also can calibrate devices, which ensures accuracy of the test data and normal operation of the devices. In short, this system's design not just satisfies the requirements of the laboratory temperature and humidity test data acquisition and monitoring alarm, also has certain theoretical significance and application value to other similar demands.

\section{Reference}

[1] Cao Mingqin,Zhang Tao,Wang Jian.Design and implementation of monitoring system for agricultural IoT based on ZigBee[J].Measurement Control Technology and Instruments,2013,39(12):

86-89.

[2] Zhang Panfeng, Zhang Jian, Wu Huali. Design of Mine Environment Monitoring System Based on ZigBee[J]. Computer Measurement \& Control, 2014, 22(11):3701-3704. 